\title{
0682 STATISTICAL MODELLING FOR RECURRENT INJURY EVENTS: AN APPLICATION TO RUGBY LEAGUE INJURIES
}

S Ullah*, C F Finch, T J Gabbett Correspondence: School of Human Movement and Sport Sciences, University of Ballarat Mount Helen Campus, University Drive, Mount Helen, P.O. Box 663 Ballarat, Victoria 3353, Australia

\subsection{6/ip.2010.029215.682}

Sports injuries can often be recurrent events, with subsequent (or repeat) injuries likely to be influenced by, and therefore different from, the first injury occurrence. While different modelling approaches have been used to analyse recurrent events, they often do not take into account correlation among the events. The aim of this study was to compare the applicability and relevance of a range of statistical models for the analysis of repeat injury count data and to provide a clear rationale for future modelling approaches for recurrent injuries. The models are demonstrated through direct application to Australian Rugby League injury data collected over three consecutive seasons. Three different models (Andersen-Gill approach, Poisson regression and negative binomial regression) were each individually fitted to the sports injury count data. Both analyti$\mathrm{cal}$ (F and bootstrap tests) and graphical approaches were used to select and compare models, including on data simulated according to various event-generating processes and differing in subject heterogeneity. Overall, the Poisson regression model provided the poorest fit to the real-world recurrent injury data and the simulated data. Although there was little difference in model fit between the remaining two models, in the interest of parsimony it is recommended that future studies routinely use the Anderson-Gill approach for the analysis of recurrent injuries. It is important that appropriate statistical methods are chosen to model recurrent injury data. To correctly specify and adequately fit recurrent injury event count data, flexible survival models should be routinely employed, with preference given to the Anderson-Gill approach. 